\title{
Oxidative stress and polycystic ovary syndrome: an evaluation during ovarian stimulation for intracytoplasmic sperm injection
}

\author{
Anderson Sanches de Melo ${ }^{1}$, Jhenifer Kliemchen Rodrigues ${ }^{1}$, Alceu Afonso Jordão Junior², \\ Rui Alberto Ferriani ${ }^{1}$ and Paula Andrea Navarro ${ }^{1}$ \\ ${ }^{1}$ Department of Obstetrics and Gynecology, Medical School of Ribeirão Preto, University of São Paulo, \\ Avenida Bandeirantes, SP, Brazil and ${ }^{2}$ Nutrition and Metabolism Laboratory, Medical School of Ribeirão Preto, \\ University of Sao Paulo, Avenida Bandeirantes, SP, Brazil \\ Correspondence should be addressed to P A Navarro; Email: drapaulanavarro@gmail.com
}

\begin{abstract}
Oxidative stress (OS) may affect the natural fertility and the results of assisted reproduction techniques (ARTs). Subfertility associated with polycystic ovary syndrome (PCOS) may be related to OS. This process may intensify during controlled ovarian stimulation (COS) for ARTs because of increased ovarian metabolic activity and hypoestrogenism with the use of gonadotropin-releasing hormone agonists (GnRHas). The objective of this study was to investigate the presence of systemic OS in non-stimulated cycles and to determine OS markers (malondialdehyde (MDA), advanced oxidation protein products (AOPP), hydroperoxides (FOX), glutathione (GSH) and vitamin E) during COS in non-obese infertile women with and without PCOS who were subjected to ARTs. A prospective cohort study was conducted on non-obese women (16 with PCOS and 60 ovulatory patients with infertility due to male and/or tubal factors). The OS markers were determined during the following time points: the follicular phase of the natural cycle (D1), after pituitary downregulation with GnRHa and before the use of gonadotropins (D2), on the day of administration of human chorionic gonadotropin (D3), and at oocyte retrieval (D4). Intergroup analysis showed that serum MDA concentrations were higher in the PCOS group at D3 $(P=0.048)$ and D4 $(P=0.002)$. On an intragroup analysis, the control group had higher MDA concentrations at D2 than at D1 $(P=0.01)$ or D4 $(P=0.004)$. The AOPP concentrations were higher at D2 $(P<0.0001), D 3(P<0.001)$ and D4 $(P<0.0001)$ compared to D1. The FOX concentrations were lower at D2 $(P<0.0001)$, D3 $(P<0.0001)$ and D4 $(P<0.001)$ than at D1. Serum GSH concentrations were significantly higher at D4 than at D1 $(P=0.02)$. An intragroup analysis of the PCOS subjects showed that the five OS markers did not differ significantly among the four time points when they were analyzed (D1, D2, D3 and D4). In conclusion, non-obese infertile women with PCOS showed evidence of systemic OS after COS with gonadotropins for ICSI. On the other hand, non-obese ovulatory infertile women and women with infertility due to male and/or tubal factors showed a possible systemic oxidative balance until the final COS.
\end{abstract}

Reproduction (2017) 153 97-105

\section{Introduction}

Polycystic ovary syndrome (PCOS) is an endocrine and reproductive disorder, ranging in prevalence between 5\% (Azziz et al. 2004) and 13\% (Melo et al. 2010) during the reproductive age. The clinical manifestations of this disorder vary according to the ethnic group and the geographic region (Wang \& Alvero 2013). Its association with infertility is common, occurring in $70 \%$ to $80 \%$ of women with the syndrome (Azziz et al. 2004). Altered folliculogenesis with the predominance of an androgenic environment and the reduction of apoptosis during the initial stages of the follicle, may promote the polycystic aspect of the ovaries and contribute to subfertility (Regan et al. 1990). It has been postulated that the worsening of oocyte quality and the impairment of endometrial receptivity may also contribute to subfertility in women with PCOS (Patel \& Carr 2008, Giudice 2006), although the mechanisms involved are still poorly understood.

The alteration of folliculogenesis in women with PCOS may be influenced by oxidative stress (OS) (Verit et al. 2007). The OS is a complex process, resulting from a disequilibrium between pro-oxidant agents (free radicals) and the antioxidant defence mechanisms of the organism. The main targets of OS are DNA, proteins and lipids. Malondialdehyde (MDA) (Petean et al. 2008) and hydroperoxides (FOX) (Kingsley et al. 1998) are products of lipid peroxidation. Oxidation and protein damage can be assessed by the determination of advanced oxidation protein products (AOPP) (Kelly et al. 2002). Conversely, vitamin E, especially in the form of alphatocopherol, is an antioxidant that can antagonize the oxidative effects of lipid peroxidation (Bornoden 1994). 
Glutathione (GSH) plays a fundamental role in peroxide neutralization and in cell protection against OS (De Matos \& Furnus 2000), participating in the processes of oocyte maturation, sperm condensation, oocyte activation and the pre-implantation development of the embryo (Agarwal et al. 2005).

Evidence has suggested that OS can affect both the natural fertility and the results of assisted reproduction techniques (ARTs) (Agarwal et al. 2012). As there is an increase in ovarian metabolic activity with controlled ovarian stimulation (COS) for assisted reproductive cycles (Yeste et al. 2016), it is possible that the production of free radicals may be increased, favoring the occurrence or worsening of OS. In contrast, the use of gonadotropinreleasing hormone agonists $(\mathrm{GnRHa})$ before $\mathrm{COS}$ for in vitro fertilisation (IVF) or intracytoplasmic sperm injection (ICSI) has been questioned because its use may decrease the serum levels of estradiol (Barash et al. 1998) and induce hypoestrogenism (Cagnacci et al. 2015); this may interfere with the oxidative balance during COS. As women with PCOS have higher antral follicle counts, an increased risk of a hyper-response to COS (Li et al. 2014) and exhibit evidence of systemic and follicular OS (Kaya et al. 2009, Kocer et al. 2014), questions have been raised regarding the occurrence of OS intensification after pituitary downregulation with GnRHa and during COS for ARTs. The evaluation of oxidative balance in women with PCOS and their possible relationship with COS for assisted reproduction could provide perspectives for new therapeutic strategies using antioxidant drugs in the presence of OS in these patients. However, to the best of our knowledge, no studies have investigated the systemic oxidative balance after acute pituitary downregulation with GnRHa and COS for assisted reproductive cycles in women with or without PCOS.

On this basis, the objectives of the present study were to investigate the presence of systemic OS in non-stimulated cycles and the role of acute pituitary downregulation with GnRHa, and the COS with gonadotropins in the systemic oxidative balance of non-obese infertile women with and without PCOS, by determining the serum concentrations of five OS markers: three pro-oxidant markers (MDA, AOPP and FOX) and two antioxidant markers (GSH and vitamin E) in the natural cycle preceding COS after pituitary downregulation with $\mathrm{GnRHa}$ and during $\mathrm{COS}$ with exogenous gonadotropins for ICSI.

\section{Patients and methods}

\section{Study design}

A prospective cohort study was conducted in the Sector of Human Reproduction at the Department of Obstetrics and Gynecology, Medical School of Ribeirão Preto (FMRPUSP), and in the Laboratory of Nutrition and Metabolism,
Department of Internal Medicine, FMRP-USP. The study was approved by the Research Ethics Committee of the University Hospital, FMRP-USP. Patients who fulfilled the inclusion criteria and who agreed to participate in the project gave written informed consent to participate in the study during their visit immediately preceding the beginning of COS.

\section{Setting and duration}

From February 2008 to January 2010, the patients who participated in the Assisted Reproduction Program of the University Hospital, FMRP-USP and had undergone COS for ICSI were evaluated according to the eligibility criteria. Those patients who were considered eligible were interviewed. Blood samples were collected: 1) during the cycle preceding COS, during the early follicular phase of the cycle for the ovulatory women or on any day of the cycle for amenorrheic women with PCOS, as long as a transvaginal ultrasonography (TVUS) did not reveal a follicle $\geq 10 \mathrm{~mm}$ or a corpus luteum (D1); 2) 10 days after pituitary blockage with GnRH agonist (D2); 3) after COS for ICSI, on the day of human chorionic gonadotropin (hCG) administration (D3) and 4) on the day of oocyte retrieval (D4). The patients were followed up until the results of ICSI were obtained. The markers under study were measured at the Laboratory of Nutrition of FMRP-USP.

\section{Participants - eligibility criteria}

The infertile women were divided into two groups: 1) PCOS: patients with PCOS diagnosed according to the Rotterdam Consensus (The Rotterdam ESHRE/ASRM-Sponsored PCOS Consensus Workshop Group 2003), and 2) Control: ovulatory patients with infertility due to only male and tubal factors. All patients underwent diagnostic video-laparoscopy as the routine procedure for the investigation of marital infertility.

The eligibility criteria in both groups were age less than 38 years, body mass index $(\mathrm{BMI})<30 \mathrm{~kg} / \mathrm{m}^{2}$, serum level of follicle-stimulating hormone $(\mathrm{FSH})<10 \mathrm{mlU} / \mathrm{mL}$ on the third day of the natural menstrual cycle, non-smoking status. The eligibility criteria also included the absence of endometriosis, hydrosalpinx, chronic diseases such as diabetes mellitus or other endocrinopathies, cardiovascular disease, dyslipidemia, systemic lupus erythematous and other rheumatologic diseases, HIV infection or any active infection. The exclusion criteria were use of hormonal medication, anti-inflammatory drugs or vitamin supplements during the six months preceding the beginning of the study or initiating the use of the above medications before or during COS or a refusal to participate in the study.

\section{Stimulation protocol}

Programming of menstruation was used to synchronize and program the beginning of the COS cycle. This procedure consisted of daily administration of combined oral contraceptives, starting during the menstrual period of the preceding cycle up to 5 days before the day scheduled for the beginning of ovarian stimulation, with their administration being discontinued in such a way that the beginning of 
menstrual bleeding would coincide with the execution of basal TVUS. This was done to observe the endometrial pattern and rules out the presence of ovarian cysts that might interfere with the response to the administration of exogenous gonadotropins or with the ultrasonographic monitoring of follicular growth.

Pituitary downregulation with a GnRHa was started the evening 10 days before the basal TVUS (long protocol) by the subcutaneous administration of leuprolide acetate (Lupron, Abbott; Reliser, Serono) at a dosage of $0.5 \mathrm{mg} /$ day (10 IU), which was maintained throughout the COS period until the day of administration of hCG (Ovidrel, Serono).

Controlled ovarian stimulation was started 5 days after discontinuing the oral contraceptive. The patients received 150-300 IU/day of recombinant FSH (FSHr) (Gonal-F, Serono; Puregon, Organon São Paulo, Brazil) subcutaneously during the first 6 days of induction. Starting on the seventh day of induction, the dose was adjusted according to follicular growth, and the endometrial thickness was monitored daily or on alternate days, by TVUS. Recombinant hCG $(250 \mu \mathrm{g}$, Ovidrel, Serono) was administered at 2200 in the presence of at least 2 follicles measuring $18 \mathrm{~mm}$ in the mean diameter. Oocytes were retrieved 34-36 $\mathrm{h}$ after the administration of recombinant hCG. Mature oocytes were subjected to ICSI 3-4 h after oocyte retrieval. Fertilization was evaluated approximately $16-18 \mathrm{~h}$ after ICSI, characterized by the presence of 2 pronuclei and 2 polar corpuscles. The embryo quality for transfer was assessed approximately 43-45 h (second day of embryo development) or 67-69 h after ICSI (third day of embryo development), based on the number and symmetry of blastomeres, percentage fragmentation and the presence or absence of multinucleation (Alpha scientists in reproductive medicine, and ESHRE special interest group of embryology 2011).

We determined the following variables: age, weight, height, body mass index (BMI), basal FSH value on the 3rd day of the natural cycle, total quantity of FSH used for COS, antral follicular count (average between right and left ovaries), the number of days of ovarian stimulation, endometrial thickness on the day of hCG administration, total number of retrieved and mature oocytes, total number of transferred embryos, fertilization rate ([number of fertilized oocytes divided by the number of oocytes injected] $\times 100$ ), cleavage rate ([number of embryos formed divided by the number of fertilized oocytes $] \times 100)$ and rate of clinical pregnancies per initiated cycle ([number of patients with embryos exhibiting a heartbeat upon TVUS 5-6 weeks after embryo transfer divided by the number of cycles] $\times 100$ ).

\section{Sample collection and processing}

Sterile vacuum tubes containing ethylenediaminetetraacetic acid (EDTA) were used to collect $5 \mathrm{~mL}$ of venous blood from the patients on D1, D2, D3 and D4. Samples were centrifuged at $1600 \mathrm{~g}$ for $10 \mathrm{~min}$, and the serum was divided into four aliquots and stored at $-80^{\circ} \mathrm{C}$ for later analysis for OS serum markers: pro-oxidants (MDA, AOPP and FOX) and antioxidants (GSH and vitamin E). All determinations were performed in duplicate by a single technician experienced in the methodologies. The technician did not have access to the experimental group's samples.

\section{Independent variables}

\section{Determination of total hydroperoxide concentration (FOX)}

The FOX test system was based on ferrous ion $\left(\mathrm{Fe}^{+2}\right)$ to ferric ion $\left(\mathrm{Fe}^{+3}\right)$ transformation by various types of peroxides in the samples analyzed. A purple-blue color complex (ferric-xylenol orange complex) was formed in the presence of ferric-xylenol, whose absorbance could be measured (Costa et al. 2006). A special reagent that was prepared on the day of the analysis (the FOX reagent) is used in this method: solution $A$ was prepared with $7.6 \mathrm{mg}$ of xylenol orange and $88 \mathrm{mg}$ of butylhydroxytoluene (BHT) in $90 \mathrm{~mL}$ of methanol; solution B was prepared with $9.8 \mathrm{mg}$ of ferrous sulfate in $10 \mathrm{~mL}$ of $250 \mathrm{mM}$ sulfuric acid $(0.13 \mathrm{~mL}$ sulfuric acid in $10 \mathrm{~mL}$ water). The two solutions were only mixed on the day of the analysis, in a 9:1 concentration. A 100- $\mu \mathrm{L}$ of serum or follicular fluid (FF), $100 \mu \mathrm{L}$ of water (for the blank) and $1 \mathrm{~mL}$ of the solution were needed for analysis, with a reaction time of $30 \mathrm{~min}$ while protected from light and at room temperature. The reaction mixture was then centrifuged at $3500 \mathrm{rpm}$ for $5 \mathrm{~min}$, and the absorbance of the supernatant was measured at $560 \mathrm{~nm}$. A curve was constructed with hydrogen peroxide at concentrations of $10-200 \mu \mathrm{M}$. The result was reported as $\mu \mathrm{mol} / \mathrm{g}$ protein.

\section{Determination of GSH concentration - total concentration of thiol or sulfhydryl groups}

Serum GSH was measured by the method originally described by Ellman (1959) and modified by $\mathrm{Hu}$ (1994), whereby thiol groups interacted with the dithionitrobenzoic acid (DTNB), forming a deep-colored anion, with a maximum peak at $412 \mathrm{~nm}$ $(\mathrm{e} 412=13,600$ per $\mathrm{M} / \mathrm{cm})$. In this assay, a $25 \mu \mathrm{L}$ aliquot of serum or FF was mixed with $1 \mathrm{~mL}$ of Tris-EDTA buffer $(25 \mathrm{nmol} / \mathrm{L}$ Trisbase, $20 \mathrm{mmol} / \mathrm{L}$ EDTA, $\mathrm{pH}$ 8.2), and an absorbance reading was taken at $412 \mathrm{~nm}$ wavelength. After this reading, a $25 \mu \mathrm{L}$ aliquot of the stock solution of DTNB $(10 \mathrm{nmol} / \mathrm{L}$ in absolute ethanol) was added to the solution, which was kept at room temperature for $15 \mathrm{~min}$. After this period, a new reading was obtained using DTNB as the blank (Costa et al. 2006). The concentration of sulfhydryl groups was calculated using GSH, and the result was reported as nmol/g protein.

\section{Determination of vitamin E concentration}

Vitamin $E$ ( $\alpha$-tocopherol) concentration in the serum was determined by the method of Arnaud et al. (1991), described as follows: A $0.5 \mathrm{~mL}$ serum sample was homogenized in $2.0 \mathrm{~mL}$ ethanol, placed in $1.0 \mathrm{~mL} n$-hexane and shaken for $2 \mathrm{~min}$. A $0.5 \mathrm{~mL}$ aliquot of the supernatant ( $n$-hexane) was carefully pipetted into a test tube and evaporated under nitrogen, suspended in $0.5 \mathrm{~mL}$ of the mobile phase consisting of acetonitrile/dichloromethanol/methanol (70:10:20, v/v/v) and filtered. A $100 \mu \mathrm{L}$ was injected into the high-performance liquid chromatography apparatus, and a reading was obtained as described below. A Shimadzu LC- $9^{A}$ apparatus was used for the determination of vitamin E concentrations using a type C-18 column (Simpack CLCODS $4.6 \times 25 \mathrm{~cm}$ ) and a $4 \mathrm{~mm} \times 1 \mathrm{~cm}$ precolumn at a flow of 
$2 \mathrm{~mL} / \mathrm{min}$. The reading was obtained by spectrophotometry with a UV-Vis at $292 \mathrm{~nm}$. The equipment was calibrated with standard alpha-tocopherol solutions (SIGMA) at concentrations of 10,20 and $200 \mu \mathrm{mol} / \mathrm{L}$. A standard alpha-tocopherol solution at a concentration of $20 \mu \mathrm{mol} / \mathrm{L}$ was injected before each reading. The equipment was programmed so that the results would be expressed as $\mu \mathrm{mol} / \mathrm{L}$ for serum.

\section{Measurement of malondialdehyde (MDA) concentration}

One $\mathrm{mL}$ amount of each sample was mixed with $2 \mathrm{~mL}$ of TCA-TBA-HCL $\quad(15 \%$ trichloroacetic acid, $0.375 \%$ thiobarbituric acid and $1.12 \mathrm{M}$ hydrochloric acid) and heated in a water bath for $15 \mathrm{~min}$. After cooling, the precipitate was centrifuged at $1000 \mathrm{~g}$ for $10 \mathrm{~min}$. The absorbance of the product was measured with a spectrophotometer (Spectronic 601-Milto Roy) at $535 \mathrm{~nm}$ wavelength. The concentrations of thiobarbituric acid reactive substances (TBARS) were calculated considering the coefficient of molar absorptivity of the product $\left(E 535=1.56 \times 10^{-5}\right.$ per $\mathrm{M} / \mathrm{cm}$ ), and the results were reported as nmol MDA/L. A standard curve was constructed using a stock solution of $10 \mathrm{mM}$ MDA prepared with tetramethoxypropane (SIGMA). The concentrations detected in the samples were under this curve, showing good linearity with the standard. The result is reported as $\mathrm{nmol} \mathrm{MDA} / \mathrm{g}$ protein.

\section{Determination of the concentration of advanced oxidation protein products (AOPPS)}

The protein oxidation products present in serum were determined by the method of Witko-Sarsat and coworkers (1996): A $40 \mu \mathrm{L}$ serum sample was mixed with $160 \mu \mathrm{L}$ of phosphate buffered saline (PBS) and $20 \mu \mathrm{L}$ of ultrapure glacial acetic acid and an absorbance reading was obtained at $340 \mathrm{~nm}$ wavelength. The curve for this reading was constructed using $10-100 \mathrm{mM}$ chloramine-T and $1.16 \mathrm{M}$ potassium iodide; $200 \mu \mathrm{L}$ of the standards were mixed with $10 \mu \mathrm{L}$ potassium iodide and $20 \mu \mathrm{L}$ acetic acid, and the plate was shaken for $6 \mathrm{~min}$ before the reading. The result was reported as $\mu \mathrm{mol} / \mathrm{L}$.

\section{Biases}

To avoid a selection bias, all patients who underwent COS for ICSI in our center and who fulfilled the eligibility criteria were invited to participate in the study. To avoid biases related to imbalances between the groups regarding important covariates related to OS and oocyte quality, highly selective eligibility criteria were adopted for patient inclusion in the study (keeping into account the relationship between some metabolic abnormalities related to PCOS and OS). Thus, the only factor related to infertility in the PCOS group was the presence of the syndrome itself. The control group presented conditions not associated with worse gamete quality or OS. The exclusion criteria were conditions potentially related to OS and oocyte quality. To avoid a performance bias, the subjects included in the study received clinical care according to the standard protocol of the service, by doctors who did not participate in the study. To avoid the detection bias regarding the determination of the different OS markers evaluated, all determinations were performed in duplicate by two technicians experienced in the methodologies. The technicians did not have access to the experimental group's samples. To avoid a reporting bias, all the results obtained were analyzed and were presented in the Results section of the present study.

\section{Study size}

Because no appropriate reported data were available to us to calculate the sample size for the five independent variables analyzed, we proposed a pilot study in which all eligible patients who agreed to participate in the study were analyzed over a recruitment period of 24 months (February 2008January 2010).

\section{Statistical analysis}

The quantitative demographic variables and the variables related to the assisted reproduction procedures were compared by the Student's $t$-test (Table 1). The qualitative variables were compared between the groups by the Fisher's exact test (Table 1). The linear mixed-effects model (random and fixed effects) adjusted using the PROC MIXED procedure was used to compare the OS markers among the days of blood collection and between the groups. The intra- and inter-group comparisons were performed using orthogonal contrast. The residual analysis was performed using normality and dispersion charts, by comparing the measured residual values and predicted values. All analyses were performed using the SAS 9.3 software, with the level of significance set at $5 \%$ $(P<0.05)$, with a power of $80 \%$.

Table 1 Demographic characteristics and variables determined before, during and after gonadotropin-controlled ovarian stimulation.

\begin{tabular}{lccr}
\hline Variable & Control $(n=57)$ & PCOS $(n=15)$ & \multicolumn{1}{c}{$\boldsymbol{P}$} \\
\hline Age $($ years $)$ & $33.0 \pm 3.9$ & $32.2 \pm 3.2$ & 0.45 \\
$\mathrm{BMI}\left(\mathrm{kg} / \mathrm{m}^{2}\right)$ & $23.7 \pm 3.3$ & $26.7 \pm 3.8$ & $<0.01$ \\
FSH $(\mu \mathrm{U} / \mathrm{mL})$ & $5.6 \pm 2.6$ & $5.3 \pm 1.7$ & 0.64 \\
Total FSH dose $(\mathrm{mlU} / \mathrm{mL})$ & $2045.1 \pm 633.0$ & $1493.8 \pm 331.1$ & $<0.01$ \\
Antral follicular count & $4.3 \pm 2.4$ & $7.1 \pm 3$ & $<0.01$ \\
Days of stimulation & $9.2 \pm 1.6$ & $8.6 \pm 1.7$ & 0.20 \\
Endometrium $(\mathrm{mm}) *$ & $10.8 \pm 2.5$ & $10.0 \pm 2.4$ & 0.28 \\
Retrieved oocytes & $6.4 \pm 3.9$ & $7.3 \pm 5.4$ & 0.44 \\
Mature oocytes & $4.9 \pm 3.4$ & $5.6 \pm 3.6$ & 0.49 \\
Fertilization rate $(\%)$ & $79.8 \pm 24.1$ & $77.1 \pm 28.8$ & 0.71 \\
Cleavage rate $(\%)$ & $88.9 \pm 19.1$ & $79.9 \pm 27.8$ & 0.15 \\
No of embryos formed & $2.6 \pm 1.4$ & $3.1 \pm 1.1$ & 0.23 \\
No of embryos transferred & $1.8 \pm 0.7$ & $1.9 \pm 0.9$ & 0.73 \\
Clinical pregnancy rate/ & $24.2(16 / 66)$ & $31.2(5 / 16)$ & 0.54 \\
$\quad$ initiated cycle $(\%)$ & & & \\
\hline
\end{tabular}

PCOS, polycystic ovary syndrome; BMI, body mass index; FSH, follicle stimulating hormone; fertilization rate, number of fertilized oocytes divided by the number of oocytes injected $\times 100$; cleavage rate, number of embryos formed divided by the number of fertilized oocytes $\times 100$; rate of clinical pregnancy per initiated cycle, number of patients with embryos exhibiting a heartbeat upon transvaginal ultrasound 5-6 weeks after embryo transfer divided by the number of cycles $\times 100$. 


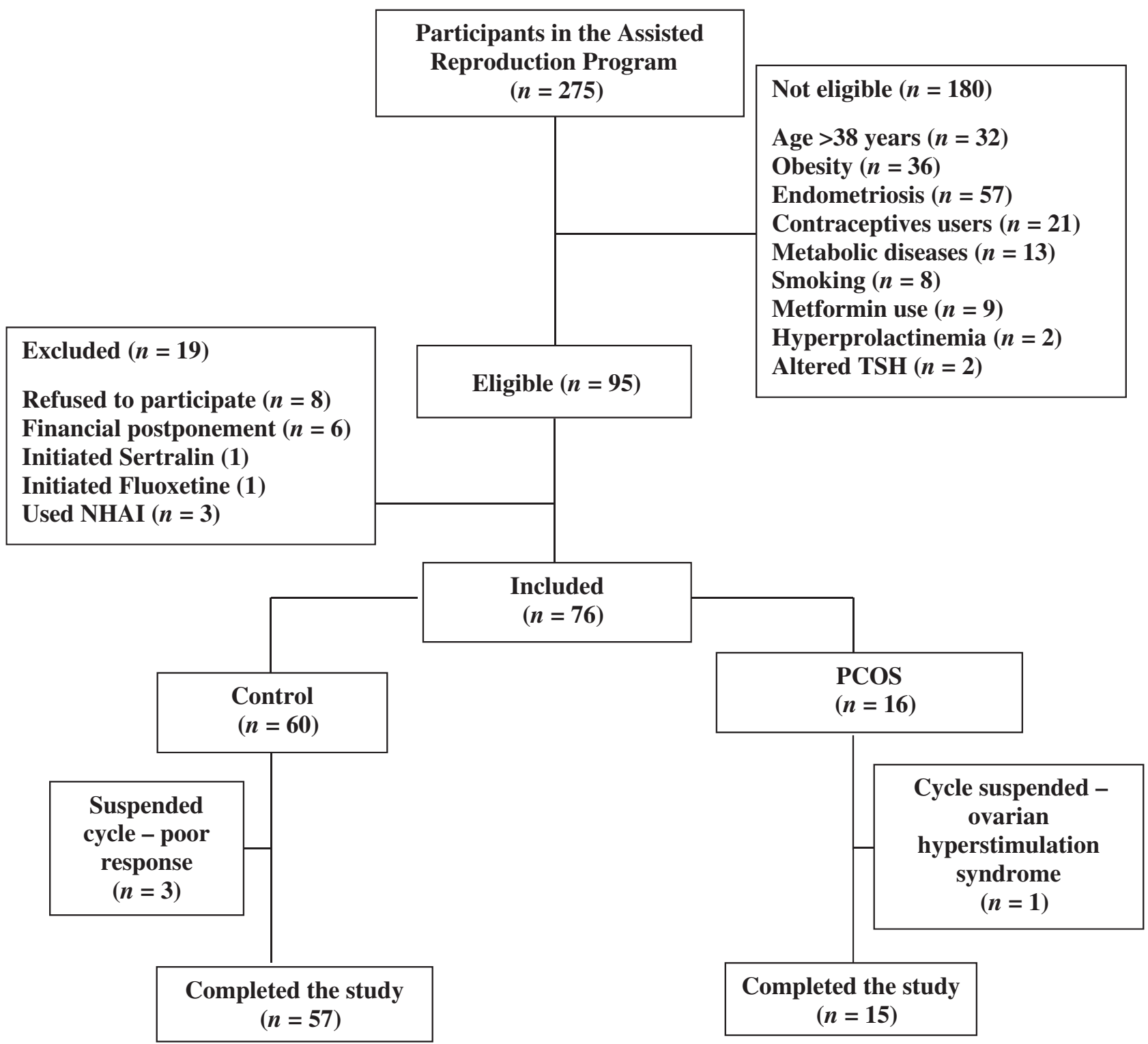

Figure 1 Flow chart of the study. PCOS, polycystic ovary syndrome; TSH, thyroid stimulating hormone; NHAI, non-steroidal anti-inflammatory.

\section{Results}

\section{Flowchart}

From October 2009 to October 2010, 275 patients attended the Assisted Reproduction Program of the University Hospital, Faculty of Medicine of Ribeirão Preto and underwent ovarian stimulation for ICSI. Of these, 180 were deemed ineligible. All 95 eligible patients (75 controls and 20 PCOS) were interviewed and 76 patients (60 controls and 16 PCOS) gave written informed consent to participate (8 patients did not agree to participate in the study, 6 patients solicited postponement of the beginning of the cycle due to financial conditions, 1 patient had started to take sertraline, 1 patient had started to take fluoxetine and 3 patients took an anti-inflammatory agent during the week preceding the beginning of COS). Three control patients discontinued due to a suspended cycle caused by a poor response, and 1 patient with PCOS discontinued due to cancellation of the cycle because of the risk of ovarian hyperstimulation syndrome (Fig. 1).

\section{Clinical variables, response to ovarian stimulation and results of ICSI for infertile patients with or without PCOS}

BMI $\left(26.7 \pm 3.8 \mathrm{~kg} / \mathrm{m}^{2}\right.$ vs $23.7 \pm 3.3 \mathrm{~kg} / \mathrm{m}^{2}$, respectively; $P<0.01)$ and antral follicular count $(4.3 \pm 2.4$ vs $7.1 \pm 3 ; P<0.01)$ were significantly higher, and total dosage of FSH used for COS was significantly lower for women with PCOS compared with the control group $(1493.8 \pm 331.1 \mathrm{mIU} / \mathrm{mL}$ vs $2045.1 \pm 633.0 \mathrm{mIU} / \mathrm{mL}$ 
respectively; $P<0.01$ ) (Table 1). There were no significant differences among the groups regarding age, basal $\mathrm{FSH}$, total FSH dose used, days of ovarian stimulation, endometrial thickness on the day of embryo transfer, number of retrieved and mature oocytes, total number of formed embryos or number of transferred embryos (Table 1). Additionally, no difference was detected in fertilization rate, cleavage rate or clinical pregnancy among the groups (Table 1).

\section{Oxidative stress markers in the serum of infertile patients with or without PCOS}

Intergroup analysis demonstrated that serum MDA concentrations were significantly higher in the PCOS group compared with the control group on D3 (PCOS: $28.7 \pm 6.9 \mu \mathrm{mol} / \mathrm{g} \quad$ vs control: $24.2 \pm 7.4 \mu \mathrm{mol} / \mathrm{g}$, respectively, $P=0.048$ ) and D4 (PCOS: $29.2 \pm 5.4 \mu \mathrm{mol} / \mathrm{g}$ vs control: $23.3 \pm 7.2 \mu \mathrm{mol} / \mathrm{g}$, respectively, $P=0.002$ ). The remaining markers did not differ significantly among the groups, at the four time points when they were analyzed (Table 2).

Intragroup analysis of PCOS subjects showed that the five OS markers did not differ significantly among the four time points when they were analyzed (D1, D2, D3 and D4) (Table 2). In the control group, serum MDA concentrations were significantly higher on D2 than on D1 (D2: $25.9 \pm 9.1 \mu \mathrm{mol} / \mathrm{g}$ vs D1: $22.6 \pm 9.0 \mu \mathrm{mol} / \mathrm{g}$, $P=0.01)$ and D4 (D2: $25.9 \pm 9.1 \mu \mathrm{mol} / \mathrm{g}$ vs D4: $23.3 \pm 7.2 \mu \mathrm{mol} / \mathrm{g}, P=0.004)$. SerumAOPPconcentrations were significantly higher on D2 $(135.1 \pm 69.7 \mu \mathrm{mol} / \mathrm{L}$, $P<0.0001)$, D3 $(118.2 \pm 63.5 \mu \mathrm{mol} / \mathrm{L}, \quad P=0.01)$ and D4 $(124.9 \pm 67.0 \mu \mathrm{mol} / \mathrm{L}, \quad P=0.0001)$ compared with those on D1 $(100.3 \pm 60.9 \mu \mathrm{mol} / \mathrm{L}, P<0.001)$. Serum FOX concentrations were significantly lower on D2 $(7.2 \pm 1.3 \mu \mathrm{mol} / \mathrm{g}, \quad P<0.0001), \quad D 3 \quad(7.1 \pm 1.3 \mu \mathrm{mol} / \mathrm{g}$, $P<0.0001)$ and D4 $\quad(7.2 \pm 1.6 \mu \mathrm{mol} / \mathrm{g}, \quad P<0.001)$ than those on D1 $(8.2 \pm 2.0 \mu \mathrm{mol} / \mathrm{g})$. Serum GSH concentrations were significantly higher on D4 than those on D1 (D1: $171.0 \pm 48.8 \mathrm{nmol} / \mathrm{L}$ vs D4: $187.0 \pm 42.6 \mathrm{nmol} / \mathrm{L}, \quad P=0.02)$. In the control group, vitamin E concentrations did not vary significantly at the time points evaluated.

\section{Discussion}

Non-obese infertile women with PCOS, when compared with infertile ovulatory women without PCOS, exhibit systemic OS, demonstrated by higher serum MDA concentrations during COS for ICSI (on the day of hCG administration and oocyte retrieval). In the present study, pituitary downregulation with GnRHa (D2) and COS with gonadotropins (D3 and D4) were not associated with changes in serum concentrations of OS markers in women with PCOS. On the other hand, non-obese ovulatory infertile women showed increased serum MDA and AOPP concentrations after pituitary downregulation with GnRHa (D2), increased AOPP concentrations during COS with gonadotropins (D2 and D3) and higher GSH concentrations in oocyte retrieval (D4). These characteristics showed that the increase in systemic oxidative markers after the use of GnRHa and COS may be counterbalanced by antioxidants (GSH) in ovulatory women. However, this oxidative equilibrium does not occur in women with PCOS, exacerbating the systemic oxidative stress in this group. Paradoxically, among the control women, the increase of MDA and AOPP levels in the serum occurred concomitantly with the reduction of FOX concentrations.

The presence of PCOS may predict the manifestation of various clinical and metabolic comorbidities (systemic arterial hypertension, type 2 diabetes mellitus and dyslipidemia) in the fourth decade of life, whereas in the healthy population, these changes are more frequent starting from the fifth decade (Elting et al. 2001, Moran et al. 2010). As these comorbidities related to PCOS may be associated with OS (Santilli et al. 2015), it is important to exclude women with these conditions to avoid bias and to improve the internal validity of our findings. Several other conditions may also be related to OS, justifying the strict eligibility criteria used in the present study and the small patient series evaluated.

Table 2 Serum markers of oxidative stress in the early follicular phase of the cycle preceding the beginning of ovarian stimulation and during ovarian stimulation with gonadotropin in the women included in the study.

\begin{tabular}{|c|c|c|c|c|c|c|c|c|}
\hline \multirow[b]{2}{*}{ Markers } & \multicolumn{4}{|c|}{ Control $(n=57)$} & \multicolumn{4}{|c|}{$\operatorname{PCOS}(n=15)$} \\
\hline & D1 & D2 & D3 & D4 & D1 & D2 & D3 & D4 \\
\hline MDA ( $\mu \mathrm{mol} / \mathrm{g} \mathrm{pt})$ & $22.6 \pm 9.0^{\#}$ & $25.9 \pm 9.1^{\#, *}$ & $24.2 \pm 7.4^{\mathrm{a}}$ & $23.3 \pm 7.2^{\mathrm{b}, *}$ & $27.9 \pm 8.9$ & $28.5 \pm 9.2$ & $28.7 \pm 6.9^{\mathrm{a}}$ & $29.2 \pm 5.4^{b}$ \\
\hline $\mathrm{AOPP}(\mu \mathrm{mol} / \mathrm{L})$ & $100.3 \pm 60.9^{\#}$ & $135.1 \pm 69.7^{\#}$ & $118.2 \pm 63.5^{\#}$ & $124.9 \pm 67.0^{\#}$ & $118.6 \pm 45.3$ & $135.3 \pm 83.9$ & $120.3 \pm 46.5$ & $121.6 \pm 76.0$ \\
\hline FOX ( $\mu \mathrm{mol} / \mathrm{g} \mathrm{pt})$ & $8.2 \pm 2.0^{\#}$ & $7.2 \pm 1.3^{\#}$ & $7.1 \pm 1.3^{\#}$ & $7.2 \pm 1.6^{\#}$ & $7.5 \pm 1.2$ & $7.9 \pm 1.3$ & $7.8 \pm 0.8$ & $7.7 \pm 1.3$ \\
\hline $\mathrm{GSH}(\mathrm{nmol} / \mathrm{L})$ & $171.0 \pm 48.8^{\#}$ & $177.1 \pm 40.9$ & $177.7 \pm 35.2$ & $187.0 \pm 42.6^{\#}$ & $188.3 \pm 58.8$ & $183.9 \pm 46.4$ & $194.3 \pm 37.3$ & $202.8 \pm 19.0$ \\
\hline Vitamin $E(\mu \mathrm{mol} / \mathrm{L})$ & $22.4 \pm 6.7$ & $22.3 \pm 5.9$ & $23.6 \pm 8.1$ & $22.7 \pm 7.3$ & $26.3 \pm 4.7$ & $25.3 \pm 11.3$ & $26.1 \pm 8.0$ & $25.1 \pm 9.2$ \\
\hline
\end{tabular}

Data are reported as mean \pm standard deviation.

Numbers followed by the same superscript on the same line indicate a statistically significant difference $(P<0.05)$. ${ }^{a, b} P<0.05$ (comparison of the control and PCOS groups).

$\#, * P<0.05$ (comparison of the serum levels of oxidative stress markers between D1, D2, D3 and D4 in the control group only).

D1, early follicular phase of the cycle preceding the beginning of ovarian stimulation; D2, day of the beginning of ovarian stimulation; D3, day of administration of human chorionic gonadotropin; D4, day of oocyte retrieval; PCOS, polycystic ovary syndrome; MDA, malondialdehyde; AOPP, advanced oxidation protein products; FOX, hydroperoxides; GSH, glutathione. 
In addition, it is known that most infertile patients with PCOS do not need to be subjected to COS for IVF and ICSI, as this is the third line of the treatment for infertility in PCOS (The Thessaloniki ESHRE/ASRM-Sponsored PCOS Consensus Workshop Group 2008), limiting the number of women eligible for the present study.

Although they included obese and non-obese women with PCOS and insulin resistance (IR), some Turkish investigators have demonstrated the presence of systemic OS in non-stimulated cycles of women with PCOS, compared with healthy women without PCOS, with OS being characterized by higher serum MDA (Kocer et al. 2014) and AOPP (Kaya et al. 2009) concentrations. Another study, also conducted in Turkey, had demonstrated increased serum MDA concentration in natural cycles of young, non-obese women with PCOS (with and without IR) compared to ovulatory women, with a positive association between MDA levels and homeostasis model assessment (HOMA) values, suggesting that $I R$ is associated with $O S$ in women with PCOS. In a previous study, higher serum MDA concentrations were detected in infertile women with PCOS (14 patients, 11 of them without IR) compared to fertile women with PCOS (15 patients, 11 of them without IR), suggesting that infertility is associated with OS in these women (Turan et al. 2015). Contrary to this, in our study, the systemic OS was associated with PCOS and not with infertility because infertile women with PCOS showed a predominance of oxidative markers without the counterbalancing of serum antioxidants during COS and oocyte retrieval, a characteristic not seen in ovulatory women.

In contrast to the present study, a cross-sectional investigation conducted in Iran reported that the increase in serum and FF levels of MDA in samples obtained on the day of oocyte retrieval from women with PCOS was not a primary characteristic of PCOS, but rather a characteristic of the high prevalence of the visceral obesity in the PCOS group. However, this Iranian study does not report the exclusion criteria (Nasiri et al. 2015), which limits the comparability of its results with the results obtained in our present study. As during follicular development in COS there is an increase in the serum concentrations of estradiol (a potent antioxidant that is normally low in natural anovulatory cycles of women with PCOS), in the present study, we were unable to demonstrate OS due to lipid peroxidation.

Among ovulatory women without PCOS, with male and tubal factor infertility, the increase in serum MDA and AOPP concentrations after pituitary downregulation with GnRHa (D2) suggests that pituitary downregulation for a short duration (10 days) may promote systemic OS. Among ovulatory women, the main sources of circulating estrogens are the dominant follicles during the first phase of the menstrual cycle, and the corpus luteum during the second phase of the cycle, which produce and release estradiol into the bloodstream (Qiao \& Feng 2011). Because hypoestrogenism may favor the occurrence of systemic OS (Cagnacci et al. 2015), pituitary downregulation with GnRHa before COS (by inhibiting the hypothalamus-pituitary-ovarian axis) reduces the serum concentrations of estradiol (Barash et al. 1998), thus favoring the occurrence of systemic OS. In this group of women, COS with gonadotropin is related to the increased serum concentrations of AOPP, suggesting that it promotes systemic OS. Paradoxically, reducing FOX concentration after the dowregulation of pituitary may be due to the fact that this oxidant serves as substrate for the formation of MDA (Pisoschi et al. 2015), which was increased after the use of GnRHa. However, the higher GSH concentrations on oocyte retrieval show that ovulatory women are able to restore the oxidative balance.

The present data do not support the modification of any current clinical protocol regarding the assessment of the systemic oxidative balance in infertile women, with or without PCOS. However, the present paper intends to trigger the discussion on the need for performing longitudinal studies to investigate the profile of OS markers during treatment for assisted reproduction, and their potential impact on the results of such treatments. If a negative impact of systemic OS on reproductive results is confirmed, it will be necessary to define the prognostic markers to be assessed and to conduct well-designed trials investigating the roles of different antioxidants in women with a diagnosis of systemic OS.

The control group was not investigated for the presence of PCOS, which could represent a limitation of the present study. However, all patients included in the control group had regular menstrual cycles associated with the perception of changes in the cervical mucus pattern, considered as sufficient data to predict ovulation in infertile women (ASRM 2012). Moreover, these women did not have hyperandrogenism symptoms.

In conclusion, non-obese infertile women with PCOS showed evidence of systemic OS characterized by higher serum MDA concentrations without counterbalancing of antioxidants markers after COS with gonadotropins for ICSI. On the other hand, non-obese ovulatory infertile women and women with infertility due to male and tubal factors showed increased serum MDA and AOPP concentrations after pituitary downregulation with GnRHa (D2), increased AOPP concentrations during $\mathrm{COS}$ with gonadotropins (D2 and D3) and higher GSH concentrations during oocyte retrieval (D4), suggesting the existence of a possible systemic oxidative balance until the final COS.

\section{Declaration of interest}

The authors declare that there is no conflict of interest that could be perceived as prejudicing the impartiality of the research reported. 


\section{Funding}

FAPESP (Grants 2008/58197-6 and 2008/52789-9), CNPq (Grant 474858/2009-0) and Instituto N acional de Ciências e Tecnologia - INCT/CNPq - Hormônios e Saúde da Mulher.

\section{Acknowledgements}

The authors wish to thank the staff of the Laboratory of Assisted Reproduction, Department of Gynecology and Obstetrics and of the Laboratory of Nutrition and Metabolism, Department of Internal Medicine, FMRP-USP, for technical support.

\section{References}

Agarwal A, Gupta S \& Sharma R 2005 Oxidative stress and its implications in female infertility - a clinician's perspective - review. Reproductive BioMedicine Online 11 641-650. (doi:10.1016/S14726483(10)61174-1)

Agarwal A, Aponte-Mellado A, Premkumar BJ, Shaman A \& Gupta S 2012 The effects of oxidative stress on female reproduction: a review. Reproductive Biology and Endocrinology 19 10-49.

Alpha Scientists in Reproductive Medicine \& ESHRE Special Interest Group of Embryology 2011 The Istanbul consensus workshop on embryo assessment: proceedings of an expert meeting. Human Reproduction 26 1270-1283. (doi:10.1093/humrep/der037)

Arnaud J, Fortis I, Blachier S, Kia D \& Favier A 1991 Simultaneous determination of retinol, alfa-tocopherol and beta-carotene in serum by isocratic high performance liquid chromatography. Journal of Chromatography 572 103-106. (doi:10.1016/0378-4347(91)80476-S)

Azziz R, Woods KS, Reyna R, Key TJ, Knochenhauer ES \& Yildiz BO 2004 The prevalence and features of the polycystic ovary syndrome in an unselected population. Journal of Clinical Endocrinology and Metabolism 89 2745-2749. (doi:10.1210/jc.2003-032046)

Barash A, Weissman A, Manor M, Milman D, Ben-Arie A \& Shoham Z 1998 Prospective evaluation of endometrial thickness as a predictor of pituitary down-regulation after gonadotropin-releasing hormone analogue administration in an in vitro fertilization program. Fertility and Sterility 69 496-499. (doi:10.1016/S0015-0282(97)00542-6)

Bornoden WR 1994 Antioxidant nutrients and protection from free radicals. In Nutritional Toxicolgy, 3rd edn, pp 19-48. Eds FN Kotsonis, N Mackey M \& JJ Hjelle. New York, USA: Raven Press.

Cagnacci A, Cannoletta M, Palma F, Bellafronte M, Romani C \& Palmieri B 2015 Relation between oxidative stress and climacteric symptoms in early postmenopausal women. Climacteric 18 631-636. (doi:10.3109/ 13697137.2014.999659)

Cohen J 1988 Statistical Power Analysis for the Behavioural Sciences. Lawrence Erlbaum Associates, Hillsdale, NJ.

Costa CM, Santos RC \& Lima ES 2006 A simple automated procedure for thiol measurement in human serum samples. Jornal Brasileiro de Patologia e Medicina Laboratorial 42 345-350. (doi:10.1590/S167624442006000100011)

De Matos DG \& Furnus CC 2000 The importance of having high glutathione (GSH) level after bovine in vitro maturation on embryo development effect of beta-mercaptoethanol, cysteine and cystine. Theriogenology 53 761-771. (doi:10.1016/S0093-691X(99)00278-2)

Elting MW, Korsen TJ, Bezemer PD \& Schoemaker J 2001 Prevalence of diabetes mellitus, hypertension and cardiac complaints in a follow-up study of a Dutch PCOS population. Human Reproduction 16 556-560. (doi:10.1093/humrep/16.3.556)

Giudice LC 2006 Endometrium in PCOS: implantation and predisposition to endocrine CA. Best Practice \& Research Clinical Endocrinology \& Metabolism 20 235-244. (doi:10.1016/j.beem.2006.03.005)

Hu ML 1994 Measurement of protein thiol groups and glutathione in plasma. Methods in Enzymology 233 380-385. (doi:10.1016/s00766879(94)33044-1)

Kaya C, Erkan AF, Cengiz SD, Dunder I, Demirel OE \& Bilgihan A 2009 Advanced oxidation protein products are increased in women with polycystic ovary syndrome: relationship with traditional and nontraditional cardiovascular risk factors in patients with polycystic ovary syndrome. Fertility and Sterility 94 1372-1377. (doi:10.1016/j. fertnstert.2008.08.016)

Kelly CJ, Speirs A, Gould GW, Petrie JR, Lyall H \& Connell JM 2002 Altered vascular function in young women with polycystic ovary syndrome. Journal of Clinical Endocrinology and Metabolism 87 742-746. (doi:10.1210/jcem.87.2.8199)

Kingsley PD, Whitin JC, Cohen HJ \& Palis J 1998 Developmental expression of extracellular glutathione peroxidase suggests antioxidant roles in deciduum, visceral yolk sac, and skin. Molecular Reproduction and Development 49 343-355. (doi:10.1002/(SICI)10982795(199804)49:4\&It;343::AID-MRD1\&gt;3.0.CO;2-N)

Kocer D, Bayram F \& Diri H 2014 The effects of metformin on endothelial dysfunction, lipid metabolism and oxidative stress in women with polycystic ovary syndrome. Gynecological Endocrinology 30 367-371. (doi:10.3109/09513590.2014.887063)

Li HW, Lee VC, Lau EY, Yeung WS, Ho PC \& Ng EH 2014 Cumulative live-birth rate in women with polycystic ovary syndrome or isolated polycystic ovaries undergoing in-vitro fertilisation treatment. Journal of Assisted Reproduction and Genetics 31 205-211. (doi:10.1007/s10815013-0151-6)

Melo AS, Vieira CS, Barbieri MA, Rosa-e-Silva AC, Silva AA, Cardoso VC, Reis RM, Ferriani RA, Silva-de-Sá MF \& Betiol H 2010 High prevalence of polycystic ovary syndrome in women born small for gestational age. Human Reproduction 25 2124-2131. (doi:10.1093/humrep/deq162)

Moran LJ, Misso ML, Wild RA \& Norman RJ 2010 Impaired glucose tolerance, type 2 diabetes and metabolic syndrome in polycystic ovary syndrome: a systematic review and meta-analysis. Human Reproduction Update 16 347-363. (doi:10.1093/humupd/dmq001)

Nasiri N, Moini A, Eftekhari-Yazdi P, Karimian L, Salman-Yazdi R, Zolfaghari Z \& Arabipoor A 2015 Abdominal obesity can induce both systemic and follicular fluid oxidative stress independent from polycystic ovary syndrome. European Journal of Obstetrics \& Gynecology and Reproductive Biology 184 112-116. (doi:10.1016/j.ejogrb.2014.11.008)

Patel SS \& Carr BR 2008 Oocyte quality in adult polycystic ovary syndrome. Seminars in Reproductive Medicine 26 196-203. (doi:10.1 055/s-2008-1042958)

Petean CC, Ferriani RA, Dos Reis RM, Dias de Moura M, Jordão AA Jr \& Andrea de Albuquerque Salles Navarro P 2008 Lipid peroxidation and vitamin $\mathrm{E}$ in serum and follicular fluid of infertile women with peritoneal endometriosis submitted to controlled ovarian hyperstimulation: a pilot study. Fertility and Sterility 90 2080-2085. (doi:10.1016/j. fertnstert.2007.10.072)

Pisoschi AM \& Pop A 2015 The role of antioxidants in the chemistry of oxidative stress: a review. European Journal of Medicinal Chemistry 97 55-74. (doi:10.1016/j.ejmech.2015.04.040)

Practice Committee of American Society for Reproductive Medicine 2012 Diagnostic evaluation of the infertile female: a committee opinion. Fertility and Sterility 98 302-307. (doi:10.1016/j. fertnstert.2012.05.032)

Qiao J \& Feng HL 2011 Extra- and intra-ovarian factors in polycystic ovary syndrome: impact on oocyte maturation and embryo developmental competence. Human Reproduction Update 17 17-33. (doi:10.1093/ humupd/dmq032)

Regan LE, Owen EJ \& Jacobs HS 1990 Hypersecretion of luteinising hormone, infertility, and miscarriage. Lancet 336 1141-1144. (doi:10.1016/0140-6736(90)92765-A)

Santilli F, D'Ardes D \& Davì G 2015 Oxidative stress in chronic vascular disease: from prediction to prevention. Vascular Pharmacology 74 23-37. (doi:10.1016/j.vph.2015.09.003)

The Rotterdam ESHRE/ASRM-Sponsored PCOS Consensus Workshop Group 2004 Revised 2003 consensus on diagnostic criteria and longterm health risks related to polycystic ovary syndrome (PCOS). Human Reproduction 19 41-47. (doi:10.1093/humrep/deh098)

The Thessaloniki ESHRE/ASRM-Sponsored PCOS Consensus Workshop Group 2008 Consensus on infertility treatment related to polycystic ovary syndrome. Fertility and Sterility 89 505-522. (doi:10.1016/j. fertnstert.2007.09.041)

Turan V, Sezer ED, Zeybek B \& Sendag F 2015 Infertility and the presence of insulin resistance are associated with increased oxidative stress in young, non-obese Turkish women with polycystic ovary syndrome. 
Journal of Pediatric and Adolescent Gynecology 28 119-123. (doi:10.1016/j.jpag.2014.05.003)

Verit FF, Erel O \& Kocyigit A 2007 Association of increased total antioxidant capacity and anovulation in nonobese infertile patients with clomiphene citrate-resistant polycystic ovary syndrome. Fertility and Sterility $\mathbf{8 8}$ 418-424. (doi:10.1016/j.fertnstert.2006.11.172)

Wang S \& Alvero R 2013 Racial and ethnic differences in physiology and clinical symptoms of polycystic ovary syndrome. Seminars in Reproductive Medicine 31 365-369. (doi:10.1055/s-0033-1348895)

Witko-Sarsat V, Friedlander M, Capeillère-Blandin C, Nguyen-Khoa T, Nguyen AT, Zingraff J, Jungers P \& Descamps-Latscha B 1996 Advanced oxidation protein products as a novel marker of oxidative stress in uremia. Kidney International 49 1304-1313. (doi:10.1038/ki.1996.186)
Yeste M, Jones C, Amdani SN, Patel S \& Coward K 2016 Oocyte activation deficiency: a role for an oocyte contribution? Human Reproduction Update 22 23-47. (doi:10.1093/humupd/dmv040)

Received 15 February 2016

First decision 21 April 2016

Revised manuscript received 29 August 2016

Accepted 26 October 2016 